\section{VULNERABILIDAD SOCIOAMBIENTAL DESDE LOS FEMINISMOS COMUNITARIOS: EL DESASTRE POR EL SISMO DEL 19S EN TETELA DEL VOLCÁN, MORELOS, MÉXICO}

Alejandra M. González-Ramírez ${ }^{1}$, Frida Güiza ${ }^{2 *}$, Martha Shirley Reyes-Quintero ${ }^{3}$, María Elena Méndez-López ${ }^{4}$, Pablo Torres-Lima 5

\section{RESUMEN}

El objetivo de este artículo es construir puentes de diálogo entre el marco de análisis de la vulnerabilidad socioambiental proveniente de la ecología política y los feminismos comunitarios de las Epistemologías del Sur, con el fin de proponer perspectivas latinoamericanas que contribuyan con la decolonización del enfoque analítico eurocéntrico de las catástrofes. El caso de estudio analiza los procesos socioambientales constitutivos del desastre por el sismo del 19 de septiembre de 2017 (19s) enuna comunidad campesina del Estado de Morelos. La metodología cualitativa incluyó la inmersión inicial en la comunidad los días posteriores a la emergencia, observación participante, entrevistas y diálogos informales. De acuerdo con los resultados, los feminismos comunitarios nombran las violencias estructurales que emergen durante la catástrofe, construidas históricamente sobre el cuerpo de las mujeres y la naturaleza; además de visibilizar el trabajo comunal de las mujeres para la atención y el cuidado en la práctica cotidiana. Destaca la Comunidad como categoría política de acción, resistencia y emancipación en el cuerpo-territoriotierra que conlleve a la disminución de su vulnerabilidad socioambiental ante el riesgo de desastre.

\section{PALABRAS CLAVES}

Desastres, Vulnerabilidad socioambiental, Feminismos comunitarios, Decolonización, Latinoamérica

\section{SOCIO-ENVIRONMENTAL VULNERABILITY FROM COMMUNITY FEMINISMS: THE DISASTER CAUSED BY THE 19S EARTHQUAKE IN TETELA DEL VOLCAN, MORELOS, MEXICO}

\section{ABSTRACT}

The aim of this paper is to build dialogues among the socio-environmental vulnerability framework influenced by the political ecology, and feminismos comunitarios' framework based on Epistemologias del Sur to decolonise the western disasters approach in the Global South. This research is based on a disaster that took place in a Mexican peasant community on September 19th, 2017. Our case study analyses the socioenvironmental processes constitutive of this disaster. Our approach was qualitative applying participant observation, in-depth interviews, and informal conversations among other techniques. Based on our results, feminismos comunitarios help to uncover historic violent practices, built over women's bodies and Nature. This framework highlights the relevance of women's communitarian work providing care and support to the whole community. Based on Feminismos comunitarios' category: body-territory-land, the idea of Community is a political category based on: action, resistance and emancipation, which might lead to address vulnerability to disasters with a different perspective.

\section{KEYWORDS}

Disaster, Socio-environmental vulnerability, Feminismos comunitarios, Decolonization, Latin America
1. Posgrado en Ciencias de la Sostenibilidad, Universidad Nacional Autónoma de México UNAM, Ciudad de México, México.

2. Investigadora independiente, Ciudad de México, México.

3. Escuela de Estudios Superiores de Tetela del Volcán, Universidad Autónoma del Estado de Morelos, Cuernavaca, México

4. CONACYT Centro de Investigación en Ciencias de Información Geoespacial CENTROGEO, Ciudad de México, México.

5. Departamento de Producción Agrícola y Animal, Universidad Autónoma Metropolitana, Ciudad de México, México.

*Autor de correspondencia: frida.guiza@gmail.com

DOI:

https://doi.org/10.55467/ reder.v6i1.83

\section{RECIBIDO}

19 de junio de 2021

\section{ACEPTADO}

18 de octubre de 2021

PUBLICADO

1 de enero de 2022

\section{Formato cita}

Recomendada (APA): González-Ramírez, A.M., Güiza, F., Reyes-Quintero, M.S., Méndez-López, M.E. \& Torres-Lima, P. (2022). Vulnerabilidad Socioambiental desde los Feminismos Comunitarios: El Desastre por el Sismo del 19S en Tetela del Volcán, Morelos, México. Revista de Estudios Latinoamericanos sobre Reducción del Riesgo de Desastres REDER, 6(1), 35-5o. https://doi. org/10.55467/reder.v6i1.83 


\section{INTRODUCCIÓN}

En los últimos 30 años el marco de análisis de los desastres se ha transformado para articular las interacciones entre naturaleza y sociedad, no obstante, son pocos los trabajos construidos con enfoques transdisciplinarios, decoloniales y desde la ecología política (Romero \& Romero, 2015; Bravo, 2017). Predominan dos visiones en disputa sobre los desastres, las cuales construyen ideas sobre cómo entender e intervenir en la realidad.

Por una parte, la apuesta teórica de la vulnerabilidad socioambiental aborda los desastres como procesos que se gestan a lo largo del tiempo, cuyas consecuencias revelan prácticas marcadas por las relaciones de poder y dominación sobre las sociedades y la naturaleza (Zanella et al., 2013; Cabral \& Zulaica, 2015). Por otra parte, una visión científica hegemónica asume que los desastres son el resultado de eventos físicos sobre comunidades humanas, por lo que se ponderan las dinámicas de los peligros naturales con índices probabilísticos para cuantificar el riesgo, desde esta lógica, los desastres son una condición posible de ser remediada con infraestructuras y análisis tecnocientíficos (Sun et al., 2019).

En este trabajo, la orientación teórica se construye desde la propuesta de la ecología política de la vulnerabilidad a desastres (Blaikie et al., 1994; Wisner et al., 2004), la cual permite la incorporación del pensamiento decolonial utilizando categorías como raza, clase y género ${ }^{1}$ (Espinosa, 2014), no solo como ejes de análisis, sino como factores estructurales originados por las opresiones que produjo el colonialismo (Curiel, 2014).

Desde la década de los años ochenta, la comunidad académica internacional interesada en el estudio de los desastres como procesos construidos socialmente, integra las consecuencias del modelo de desarrollo modernizador en el incremento de las desigualdades socioeconómicas, por la implementación global del modelo económico neoliberal (Escobar, 2007; Rojas, 2015). En este escenario de transformaciones socioeconómicas mundiales, también se reconocen tanto la crisis ambiental, como los efectos negativos en los grupos sociales históricamente excluidos: mujeres, migrantes, indígenas y afrodescendientes.

Respecto a los estudios de género el desarrollo teórico es abundante, aunque predominantemente anglosajón (Yadavet al., 2021; Bradshaw, 2013), además, los casos de estudio se llevan a cabo en el Sur global poniendo en evidencia que las mujeres son las más afectadas durante las crisis y emergencias (Gaillard et., 2017; Bradshaw \& Arenas, 2004).

Aunque se reconoce que las experiencias de desastres de las mujeres son un mosaico de necesidades y capacidades como lo son las numerosas culturas (Enarson \& Morrow, 1998), aún predomina una visión universalista de las mujeres ${ }^{2}$ (feminismo hegemónico) que lleva impresa la colonización discursiva (Curiel, 2017), es decir, a partir de un privilegio hegemónico (racial, de clase y geopolítico) se analiza la condición vulnerable de la mujer del Sur asumiéndola como un sujeto monolítico y ahistórico.

Ante el avasallamiento del feminismo hegemónico, del cual la academia forma parte (Galindo, 2013), se requieren aparatos teóricos alternativos que contribuyan en el análisis de realidades contextualizadas en y por el Sur (Gargallo, 2016). Una alteridad que defina ejes de opresión y posibilite el análisis de los procesos socioambientales constitutivos del desastre.

Este trabajo reflexiona sobre los vacíos teórico-metodológicos de los enfoques feministas hegemónicos occidentales de los desastres y explora las posibilidades del marco de los feminismos comunitarios (Paredes, 2017). Esta perspectiva latinoamericana antipatriarcal, antirracista y anticapitalista, aboga por formas de resistencia-defensa del territorio-tierra y nombra las opresiones histórico-estructurales sobre el cuerpo-territorio de las mujeres.

\section{MARCO TEÓRICO}

A continuación, se hace un breve recorrido por las propuestas que enriquecen la construcción de los feminismos comunitarios, en cuanto a la conceptualización de mujer, género, feminismo, comunidad y territorio. Uno de los temas centrales de la epistemología feminista en Latinoamérica, es el marco analítico de la colonialidad del poder (Quijano, 2000), la colonialidad del ser (Maldonado, 2007) y la colonialidad del saber (Lander \& Castro, 2000), los cuales develan las relaciones de opresión/subordinación de los sujetos/identidades sociales, que resisten la violenta imposición hegemónica del patrón occidental a partir del hecho colonial de 1492.

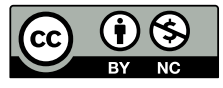

Todos los artículos publicados en REDER siguen una política de Acceso

Abierto y se respaldan en una Licencia CreativeCommons Atribución-NoComercial 4.0 Internacional.

Revista de Estudios Latinoamericanos sobre Reducción del Riesgo de Desastres (REDER)

Diseño: Lupe Bezzina

El género como concepto y categorí política denunciatoria devela la posición de subordinación de las mujeres.

2. El universalismo de género es la presunción de que todas las mujeres tienen las mismas problemáticas, reclamos y que todas tienen una sola lucha contra el patriarcado. 
Lugones (2008), teórica feminista decolonial, resignifica el marco de la colonialidad del poder al considerar que no aborda de manera precisa el papel de las mujeres en la constitución del poder colonial. Propone la idea del sistema moderno/colonial/género, el cual contribuye a reconocer y nombrar las formas de exclusión que las mujeres no occidentales viven dentro del sistema eurocéntrico global.

Los debates del feminismo decolonial incluyen teóricas feministas afrocaribeñas y negras, para quienes es relevante el eje analítico mujer-raza (Espinosa, 2014; Curiel \& Galindo, 2015), así como la imbricación de sujetos sociales, en una matriz de dominación (Hill, 1998), la cual rebasa la idea de interseccionalidad y asigna un carácter práctico/político (praxis). Por su parte, las feministas autónomas cuestionan el doble discurso europeo y neocolonial constituyente de las ideas de desarrollo, progreso y modernidad promovido por las instituciones, el Estado y las ONGs (Galindo, 2013).

También algunas mujeres indígenas se afirman abiertamente feministas, desde un pensamiento autónomo como el feminismo comunitario (Paredes, 2013); en contraste, otras mujeres indígenas no se autodenominan feministas por considerarle una categoría occidental, blanca y urbana (Gargallo, 2014). Nutrido con estos debates, se construye el feminismo comunitario como teoría social de las voces históricamente marginadas y subalternas, cuyo objetivo es la constitución de una praxis situada en una realidad que afirme la autonomía de los pueblos y reconozca las diversas opresiones de género en los cuerpos y en los territorios (Moore, 2018).

Lorena Cabnal, maya K'iche' (2017; 2012), menciona que esta propuesta nace dentro de lo cotidiano, desde un territorio-cuerpo-tierra indignado por problemáticas sociales-ambientales; se construye como re-creación del pensamiento cosmogónico feminista para reinterpretar realidades de la vida de las mujeres indígenas. Tranquilina Morales, del pueblo Me'phaa (Guerrero, México), señala que el feminismo comunitario es espacio de encuentro, de fraternidad y solidaridad con las mujeres y con la mitad del universo social, el hombre (Berrio, 2019).

El feminismo comunitario por su constitución histórica y social, cuestiona la visión binaria dominante en los feminismos hegemónicos también llamados de la diferencia, y propone los principios de dualidad, complementariedad y equilibrio, estableciendo una relación de completud e interdependencia (Paredes \& Guzmán, 2014), entre hombres y mujeres, y entre naturalezasociedad. Por otro lado, pone en duda el universalismo occidental justificado con el discurso de equidad de género, y se señala como un concepto superficial y descriptivo de roles más que denunciatorio de la subordinación (Paredes, 2013).

Con esta mirada, el feminismo comunitario se asume como la lucha de cualquier mujer, en cualquier parte del mundo y en cualquier tiempo de la historia, que se rebela al patriarcado que la oprime (Paredes, 2013; 2017). Este feminismo permite releer y reconstruir la historia de cada pueblo, a partir del reconocimiento de la lucha de las mujeres antepasadas en la vida social comunal, con una idea de dualidad cosmogónica.

Los dos elementos claves en esta propuesta feminista son, el cuerpo-territorio-tierra y el entronque patriarcal (Moore, 2018). El primero se desarrolló como consigna política de las mujeres indígenas Mayas Xincas (Guatemala) tras el avance de la violencia sexual sobre el cuerpo de las mujeres y la lucha ambiental contra la minería (Cabnal, 2017). Así, la categoría cuerpo-territoriotierra permite entender las diferentes opresiones no solamente de género, coloniales, racistas y neoliberales sobre los cuerpos y las tierras ancestrales, sino simultáneamente ubica el lugar de resistencia para lograr la emancipación de las mujeres y el respeto de la naturaleza por medio de la recuperación de la red de la vida.

El segundo, enuncia la existencia de un patriarcado originario ancestral (Paredes \& Guzmán, 2014) o patriarcado de bajo impacto (Segato, 2014), como un sistema milenario estructural de opresión contra las mujeres originarias, el cual norma la hetero-realidad como mandato para la vida de las mujeres/hombres y de ambos en su relación con el cosmos (Cabnal, 2012). Esta categoría además nombra el entronque patriarcal o penetración colonial posterior al hecho histórico de 1492, que se contrapone a otras propuestas teóricas feministas que sostienen la idea de que la raza y el género se producen simultáneamente en el proceso de conquista y colonización (Lugones, 2008; Quijano, 2000). Para las feministas comunitarias ambas raíces son las causas de que los cuerposterritorios y el territorio-tierra vivan violencias patriarcales y coloniales. 
Finalmente, es necesario destacar que el feminismo comunitario propone a la Comunidad como el eje de lucha, creadora de identidad para la acción colectiva en espacios indígenas, campesinos, rurales o urbanos, la cual emerge como proyecto político, como un horizonte de posibilidades, como una utopía que se construye (Paredes \& Guzmán, 2014) mediante procesos de organización y participación conjunta (Cueto et al, 2015).

\section{MARCO METODOLÓGICO}

Esta investigación se construyó con una perspectiva metodológica cualitativa (Strauss \& Corbin, 2016; Neuman, 2000), la cual se aplica en un estudio de caso, con un enfoque interpretativo que permite entender los múltiples significados que las personas asignan a las situaciones específicas y al espacio físico en el que viven (Geertz, 2008); esto se adscribe al marco teórico de la vulnerabilidad socioambiental (Blaikie et al., 2014) y al feminismo comunitario (Paredes \& Guzmán, 2014). La densidad en la descripción (thick description) es clave del proceso de investigación cualitativa, la cual aumenta con la observación, entrevistas a profundidad y conversaciones informales con los diferentes actores (Stake, 2005).

\section{La etapa inicial}

El proceso de investigación inició con la experiencia personal de la autora principal de este trabajo, en el sismo del 19 de septiembre de 2017 en la Ciudad de México. Algunos informantes fueron clave como el Geógrafo A, "El día 20 de septiembre, a las 20:00, el geógrafo A llamó para invitarme a ir a la comunidad de donde es originaria su mamá, en Morelos, esta no había recibido apoyo de ningún tipo y las afectaciones eran mayores" (notas de campo personales).

Gracias a este informante clave, mediante la técnica bola de nieve se contactó a otras personas de la comunidad, organizaciones no gubernamentales, académicos(as) e instituciones locales involucradas en la respuesta de la emergencia. También se estableció vínculo con la directora de la Escuela de Estudios Superiores de Tetela del Volcán - EESTEVOL (ver Tabla 1).

La inmersión inicial del 21 de septiembre al 8 de octubre de 2017 no fue influida por la perspectiva teórica, lo cual permitió vivir la experiencia como un actor externo participante en el mundo social (Corbetta, 2003). La experiencia se sistematizó posterior a la crisis, para entender las racionalidades/sentires de la gente de la comunidad, los diferentes actores externos con los cuales se tuvo contacto, así como las observaciones que se hicieron sobre el contexto del desastre, con el fin de entender cómo se construyen, negocian y operan las trayectorias de los diferentes participantes en el sitio de la emergencia.

\section{Segunda etapa}

Se hicieron 6 visitas durante 2018 y 2019 a la zona de estudio; en una de ellas se tuvo conocimiento de primera mano sobre el contexto socioambiental, prácticas agrícolas y posibles zonas de riesgo en Tetela del Volcán: "(...) se organizó una visita al volcán Popocatépetl con uno de nuestros amigos "AA" que conocimos durante la emergencia, originario de Tetela y participante activo del grupo ambiental de la comunidad. En total fuimos 7 personas, 3 geógrafos(as) externos a la comunidad y 4 biólogos(as) de Tetela del Volcán. El objetivo, fue realizar un vuelo con dron para identificar otras problemáticas ambientales de la comunidad y de su área natural protegida próxima al volcán, con miras a un ordenamiento ecológico comunitario" (Notas de campo personales).

Para un mayor acercamiento con las mujeres de Tetela del Volcán se participó en actividades desarrolladas por la EESTEVOL, como estrategias dirigidas a la recuperación económica y emocional con terapias tradicionales basadas en la herbolaria ${ }^{3}$, implementadas en un temazcal ${ }^{4}$. Previo a la declaración de emergencia sanitaria y confinamiento por COVID-19, se realizó una última visita de campo en noviembre de 2019 durante el día de muertos, una de las fiestas más importantes en México.

\section{Técnicas de recolección de datos}

El enfoque cualitativo permite de manera flexible adecuarse a los cambios y situaciones que se presentan (Strauss \& Corbin, 2016). Por consiguiente, la última etapa consistió en la revisión de fuentes documentales con perspectivas diversas relacionadas con el tema de estudio, tales como documentos gubernamentales, censos y planes de desarrollo municipales, cartografía, atlas de riesgos, periódicos locales, artículos científicos, libros y tesis referentes al sitio de estudio. La información disponible sobre esta localidad específica consistió en la revisión de cuatro libros, nueve tesis y cinco artículos científicos.
3. Actividad de la medicina tradicional mexicana que hace uso de las plantas y animales para recuperar la salud física, mental y espiritual.

4. El temazcal es un ritual ancestral que incluye el uso del vapor de agua con hierbas medicinales en un espacio cerrado, su misión es la sanación y limpieza del cuerpo espiritual y físico. 
Una de las técnicas principales de recolección de datos primarios fue la observación participante, que permitió tener una visión profunda de las dinámicas y procesos sociales que se llevan a cabo en Tetela del Volcán, información que fue posteriormente analizada con una mirada feminista (Castañeda, 2012). La observación reflexiva y crítica de este espacio, permitió captar algunos significados de la cultura, formas de organización y el sentido que otorgan las personas a sus acciones y las prácticas que reproducen la opresión de las mujeres dentro de las comunidades (Serrano, 2013; Moore, 2018).

Estas observaciones fueron hechas de manera directa, tratando de aprehender aspectos específicos del contexto como roles, características del espacio físico, relaciones entre personas, también de manera indirecta, participando en diferentes actividades como el temazcal, la fiesta del pueblo o bien las actividades posteriores a la emergencia. Todas las observaciones fueron hechas como notas y borradores, las cuales fueron sistematizadas en una gran narración que permitió establecer relaciones, procesos e interacciones en la comunidad.

El temazcal como espacio colectivo donde hombres y mujeres participan permitió conocer temas de preocupación de la comunidad, tanto posteriores al desastre como en la vida cotidiana, además de ahondar a través de una práctica corporal, en situaciones traumáticas, dolorosas y delicadas (Acuña, 2016; Cabnal, 2017) con la visión holística característica de los pueblos originarios de América Latina.

Los principales temas de las entrevistas fueron: Contexto local; Mujeres y violencias; Cultura y poder; Problemáticas socioambientales; y Peligros socionaturales/19S. En la tabla 1, se presentan los temas, las personas y lugares de presencia de las/los entrevistados.

\begin{tabular}{llll} 
Actores y lugar de procedencia & Edad aprox. & Sexo & Tema \\
\hline Geógrafo A (Tetela del Volcán/CDMX) & 25 & $\mathrm{H}$ & Contexto local \\
\hline Directora EESTEVOL-UAEM (Tetela del Volcán) & 42 & $\mathrm{M}$ & Cultura y poder \\
\hline Mujer F (Tetela del Volcán) & 53 & $\mathrm{M}$ & Afectaciones 19S \\
\hline $\begin{array}{l}\text { Grupos de evaluación-Universidad UMSNH } \\
\text { (Michoacán, México) }\end{array}$ & $22-26$ & $\mathrm{H}$ & $\begin{array}{l}\text { Problemática } \\
\text { socioambiental }\end{array}$ \\
\hline $\begin{array}{l}\text { Estudiantes de Universidades públicas y privadas } \\
\text { IBERO, IPN, UAM, UNAM (CDMX) }\end{array}$ & $18-30$ & $\mathrm{H} / \mathrm{M}$ & $\begin{array}{l}\text { Peligros } \\
\text { socionaturales }\end{array}$ \\
\hline Mujeres del Temazcal (Tetela del Volcán) & $17-60$ & $\mathrm{M}$ & Mujeres/violencias \\
\hline ONGS AA (Tetela del Volcán) & 39 & $\mathrm{H}$ & Contexto local \\
\hline
\end{tabular}

Tabla 1: Temáticas de entrevistas y diálogos informales. Fuente: Autoras, 2022.

\section{EL CONTEXTO LOCAL DE TETELA DEL VOLCÁN}

Tetela del Volcán es un municipio localizado al noreste del Estado de Morelos, a $50 \mathrm{~km}$ de la Ciudad de México (CDMX) (ver Figura 1). La ubicación del estado favorece el intercambio y movilidad de mercancías y personas con la capital del país, funciona también como espacio descentralizador de actividades y servicios (Martínez, 2008). En el transcurso de su historia, esta entidad ha transitado por diferentes etapas desde el desmantelamiento del sector agropecuario, la terciarización de la economía, la segregación espacial por grandes cadenas inmobiliarias, la marginación de comunidades indígenas y la implementación de megaproyectos como el Plan Integral Morelos (termoeléctricas). Morelos posee una larga historia de marginación, violencia estructural y conflictos socioambientales, que colocan a 50,8\% de la población en situación de pobreza (CONEVAL, 2015).

En este contexto, Tetela del Volcán tiene una dinámica interna influenciada por las tradiciones indígenas, campesinas y religiosas en donde coexisten dos formas de gobierno, el municipio y los usos y costumbres; ambos autogestionan los bienes de uso común (volcán, bosques, agua, tierra) y la toma de decisiones que se hace través de las asambleas del pueblo (Reyes, 2011 y 2017). 


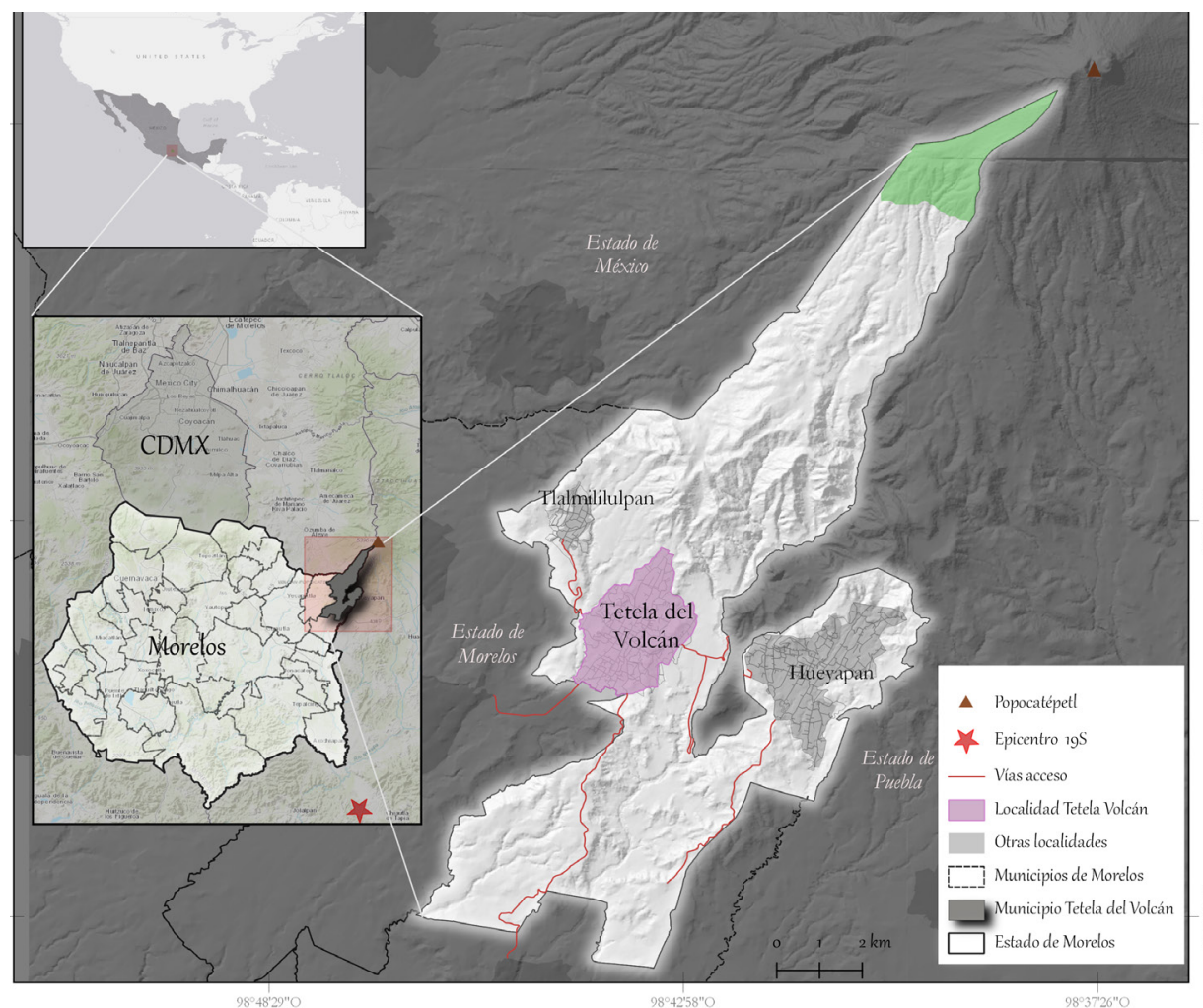

Figura 1. Localización de Tetela del Volcán Morelos

Fuente: Autoras, 2022, con base en INEGI, información vectorial, 2010

Sus elementos biofísicos como la cercanía al volcán Popocatépetl, disponibilidad de agua dulce proveniente de los deshielos del volcán, la fertilidad del suelo, el relieve accidentado, el clima templado y su vegetación abundante (Reyes, 2017) hacen de la agricultura campesina la principal actividad económica del municipio. Según datos de la Secretaría de Desarrollo Social (SEDESOL) los peligros socio naturales consisten en heladas, fracturas geológicas, deslizamientos y derrumbes, peligro sísmico y volcánico tanto por flujos de materiales como por flujos de lahares (SEDESOL, 2012).

En 2015, la población total en el Municipio de Tetela del Volcán fue de 20.698 personas, de ellas 10.074 hombres y 10.062 mujeres; $47,6 \%$ de la población del municipio con autoadscripción indígena Nahua (INEGI, 2015). El total de hogares fue de 4.882, ocupados mayoritariamente por composiciones familiares nucleares y ampliadas, con un promedio de 4.2 personas por vivienda, el más alto de Morelos (INEGI, 2015). Para este mismo año, el 81,4\% de la población se encontró en situación de pobreza, el segundo lugar del Estado de Morelos y con más de una carencia social (ver Tabla 2).

\begin{tabular}{lcc} 
Tipo de carencia & $\mathbf{2 0 1 0} \%$ & $\mathbf{2 0 1 5} \%$ \\
\hline Acceso a servicios de salud & 46.4 & 14.0 \\
\hline Acceso a seguridad social & 90.8 & $\mathbf{9 0 . 9}$ \\
\hline Calidad y espacios de vivienda & 25.3 & 17.4 \\
\hline Acceso a los servicios básicos en la vivienda & 51.9 & $\mathbf{6 3 . 9}$ \\
\hline Acceso a la alimentación & 13.0 & $\mathbf{1 6 . 7}$ \\
\hline
\end{tabular}

Tabla 2: Porcentaje de población de acuerdo al tipo de carencia social

Fuente: Autoras, 2022, con base en la Comisión Nacional de Evaluación de la Política de Desarrollo Social (CONEVAL, 2015).

\section{El desastre por el sismo del 19s. Peligros Socionaturales}

En México, a las 13:14 hora local del martes 19 de septiembre de 2017 se registró un sismo de 7.1 grados con epicentro en los límites del Estado de Morelos y el Estado de Puebla, a $120 \mathrm{~km}$ de la CDMX (SSN, 2017), este ocurrió dentro de la placa oceánica de Cocos por debajo del continente a una profundidad de $57 \mathrm{~km}$. El Centro Nacional de Prevención de Desastres (CENAPRED) 
reporta que fue uno de los sismos con mayores afectaciones en Ciudad de México, Estado de Morelos y Estado de Puebla por su magnitud, su duración, cercanía del epicentro, aceleraciones máximas alcanzadas y por el monto de pérdidas y daños evaluado en 62.099 millones de pesos en afectaciones y 369 pérdidas humanas (CENAPRED, 2019).

Tetela del Volcán encabezó la lista de municipios de Morelos con mayor porcentaje de daños en las viviendas afectadas $(29,5 \%)$ y de planteles educativos (91\%) (Gobierno del Estado de Morelos, 2017). De acuerdo con la información recabada con autoridades municipales, recorridos en campo e indagación local, las afectaciones fueron muy extendidas, con pérdidas humanas de dos mujeres fallecidas (ARP Prensa, 2017), daños materiales a infraestructura hidráulica (Gobierno del Estado de Morelos, 2017), infraestructura de comunicaciones, carreteras y vialidades urbanas dañadas(ARP Prensa, 2017), afectaciones ambientales y cambios en el territorio por apertura de grietas y procesos de remoción en masa con 20 viviendas afectadas (Barranco, 2018), daños en parcelas de cultivos con caídas de árboles frutales y deterioros estructurales en edificios históricos del periodo colonial.

El gobierno del Estado de Morelos (2017) reportó además que 20,5\% de las unidades económicas resultaron afectadas, así como 1.441 viviendas, de ellas 629 sufrieron daños totales, muchas de ellas de arquitectura vernácula (adobe). Durante los recorridos en campo se observó a decenas de personas que se quedaron a la intemperie, en improvisadas carpas o techumbres con plásticos, lonas, láminas de cartón, sujetados a postes de madera por el miedo de acudir a los albergues y perder las pocas pertenencias.

\section{RESULTADOS}

\section{La tierra: Agricultura y transformaciones comunitarias violentas}

El sismo en Tetela del Volcán evidenció algunas consecuencias de la entrada de las reformas neoliberales implementadas en el país a finales del siglo pasado (Varela, 1985), específicamente en las transformaciones sobre el territorio, en las formas de subsistencia de la población y en la organización interna de la comunidad. Las iniciativas de reajuste fiscal de los Estados (Fini, 2016), la privatización de derechos sociales (vivienda, salud, educación), el recorte presupuestario en los estados, así como la municipalización, fueron presiones que debilitaron las formas de gobierno comunal, específicamente la Asamblea de Pueblo como forma de elegir a las o los gobernantes de Tetela del Volcán.

Asimismo, las tierras ejidales y comunales para el cultivo agrícola y frutícola de temporal se transformaron dramáticamente en la década de 1980 con la propuesta del presidente municipal y gobernador del Estado, para tecnificar el campo y promover el desarrollo. Con ello se transformó la agricultura de subsistencia de temporal por la intensiva del riego basado en la extracción de agua de manantiales del Volcán Popocatépetl, como de manantiales a flor de tierra y de la microcuenca del río Amatzinac (Reyes, 2011).

Desde una escala macro, la tierra se vio amenazada mediante la desvalorización de los espacios rurales, la mercantilización de los bienes comunes y la globalización del sistema agroalimentario (Martínez, 2008), con efectos negativos locales como la contaminación del agua y suelo por agroquímicos, el uso desmedido de agua de río y manantiales para riego, la sobresaturación de suelo y la deforestación del bosque nativo. Además del surgimiento de problemáticas socioeconómicas en los campos agrícolas como su dependencia a insumos químicos y la conversión de un sistema productivo local a uno dependiente del mercado regional principalmente a través del cultivo de aguacate, lo cual, dio pauta al aumento del cacicazgo de intermediarios para la comercialización de los productos del campo a mayor escala (Bazaldúa \& García, 2014).

Este cambio trae como consecuencia un ingreso mayor, pero no mejor, porque no son pagos justos dado el intermediarismo que existe para la venta de sus productos. Al respecto, en una entrevista con la directora del EESTEVOL, describe cómo estos cambios graduales, no han mejorado la calidad de vida de las familias y evidencia la tendencia a la descampesinización de las nuevas generaciones:

"Los campesinos en su mayoría adultos mayores de 60 años, no ven una necesidad de tener más. Sin embargo, hay un desfase generacional desde hace aproximadamente 20 
años con los jóvenes que no desean acceder a trabajar la tierra, ya no buscan acceder a lo mínimo, entonces se visibiliza la pobreza relacionada con la escasez y los bienes materiales. Cambia la percepción de ser pobre" (Directora EESTEVOL).

Esta perspectiva sobre la pobreza se evidencia en Tetela del Volcán por las aspiraciones de migrar hacia las ciudades cercanas, para acceder a trabajos urbanos y a los servicios educativos que son limitados en la comunidad. También se expresa en la transformación de las viviendas tradicionales, pues el adobe (arcilla), material principal de la arquitectura vernácula de la región se vio desplazado por materiales como concreto, block y cemento. Ante la necesidad de mostrar una imagen de modernidad, la falta de apropiación a nuevos sistemas constructivos, la falta de una normatividad de construcción y en otros casos la ampliación de las viviendas por el incremento de las familias aumentó el riesgo debido a la falta de compatibilidad de materiales usados, "Durante el recorrido de la comunidad fue común encontrar primeros pisos de adobe y segundos de concreto" (Notas de campo personales).

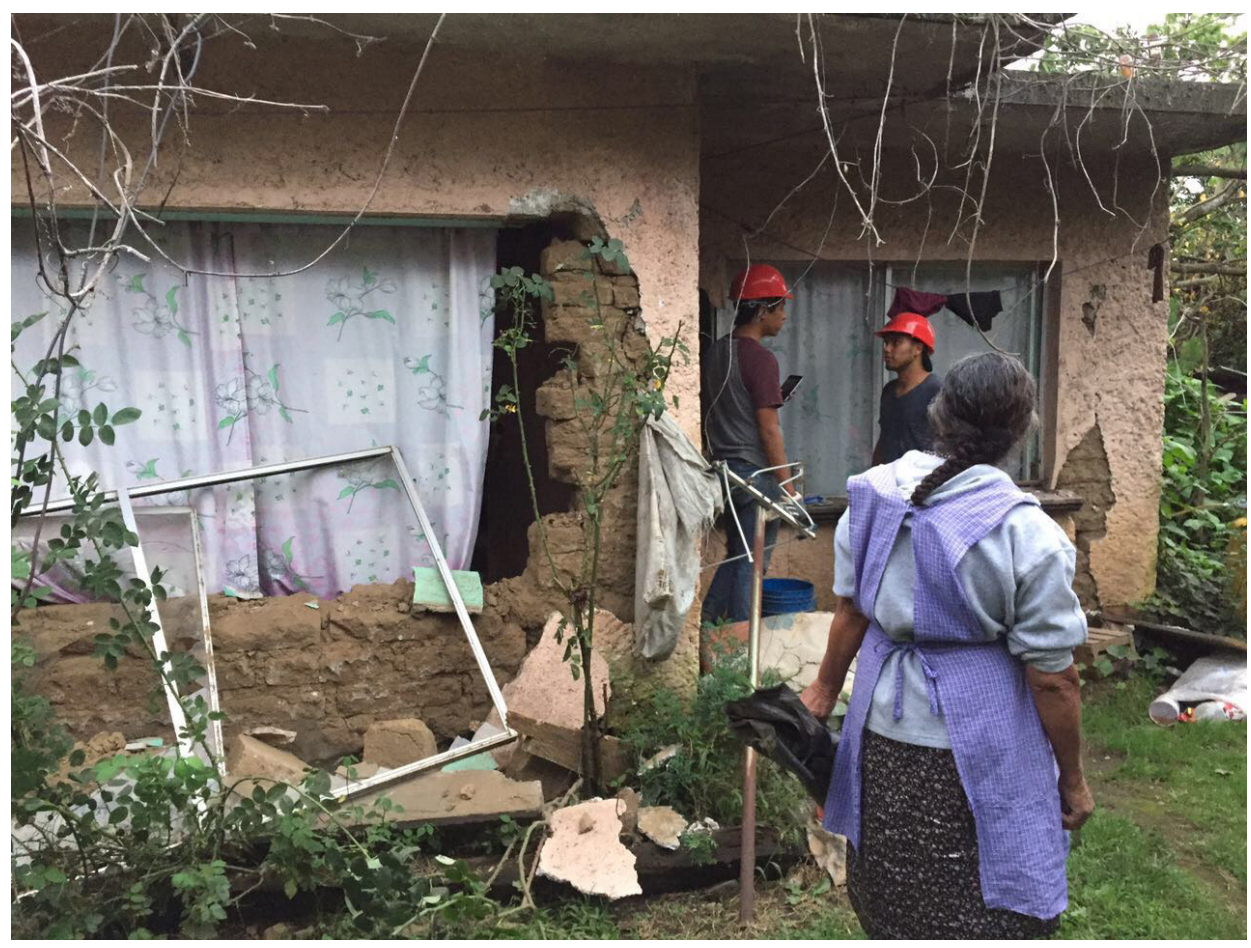

Figura. 2. Daños en viviendas vernáculas durante el 19s modificadas con nuevos materiales en Tetela del Volcán Fuente: Fuente: Autoras, septiembre de 2017

Asimismo, se pudo percibir durante las entrevistas y conversaciones con evaluadores externos provenientes de universidades, que muchas viviendas se encuentran en espacios de alto riesgo como laderas de cerros, a orillas de las barrancas o en suelos inestables, lo que incrementa el riesgo de las familias ante la amenaza de desastres por sismos, procesos de remoción en masa o erupción volcánica.

\section{Cuerpo-Territorio: Violencia social y doméstica}

En relación con la violencia social y doméstica como componentes de la vulnerabilidad, los hallazgos de la investigación muestran que en Tetela del Volcán algunas tradiciones, costumbres y prácticas cotidianas desencadenan en las mujeres la invisibilización de su trabajo doméstico y comunitario; el control sobre el cuerpo; la división sexual y racial del trabajo; la segregación espacial; la dependencia económica, emocional y patrimonial hacia la figura masculina; y el olvido de sus saberes ancestrales.

En las conversaciones con mujeres dentro del temazcal se pudo corroborar la prevalencia de la idea de la conformación de la familia desde muy temprana edad, frecuentemente encontrada en las comunidades campesinas e indígenas (Tzul, 2015), así como su papel de organizador social de la vida: "Las mujeres más jóvenes tuvieron mayor dificultad para hablar y expresar sus emociones fácilmente, pero mencionaron lo difícil que es no poder vivir libremente, tomando sus 
propias decisiones sin el peso del qué dirán, sobre todo la familia" (Notas de campo personales). El carácter patrilineal de la herencia de las tierras se suscita mediante lazos de parentesco, el matrimonio facilita que las familias recién formadas, y de manera indirecta las mujeres pueden tener acceso a tierras o viviendas, ingresos económicos y prestigio social frente a la sociedad (González, 2019; Reyes, 2017). En la localidad, los jóvenes varones adultos que forman una familia, reciben como herencia una porción de la parcela familiar de parte del padre, en tanto que las mujeres 'acceden' a la tierra a través de sus esposos 0 al enviudar, aunque siempre con el respaldo del hijo varón (Reyes, 2017).

Las responsabilidades dentro del hogar, es decir trabajo no remunerado, implican en promedio 55.8 horas a la semana (INEGI, 2015), respecto a 17 horas en promedio de los varones, sin embargo, las posibilidades de las mujeres para incidir políticamente en las decisiones de la comunidad son pocas. Situación que se agudiza en mujeres de la tercera edad, mujeres embarazadas, mujeres con discapacidad, mujeres con dependientes económicos, o mujeres que tienen que salir a trabajar para mantenerse ellas mismas.

Otra violencia más sutil que nuestros datos confirman, es el silenciamiento de mujeres para la toma de decisiones en todos los niveles. Los jefes de familia suelen beneficiarse del manejo de la tierra, aunque la esposa o hijas contribuyan con el trabajo, generalmente es él quien decide qué se siembra, cómo se vende y en qué se gastan los ingresos económicos de la siembra:

"El varón siempre tiene todos los privilegios desde las tierras y los medios de producción, de hecho, si las mujeres desean dar estudios a sus hijos, ellas convencen primero al esposo para dar permiso de que puedan estudiar, y ellas se hacen cargo de los gastos" (Directora EESTEVOL).

De acuerdo con la información recolectada, respecto a la participación política en espacios comunitarios, desde hace 15 años aumentó la presencia de mujeres mayordomas (organizadoras de los eventos religiosos) y dentro de otras organizaciones como las cajas de agua, agrupaciones del mercado municipal, entre otras, sin embargo, su papel de organizadoras no es reconocido ni valorado.

Así, las pocas mujeres que pueden participar o tomar decisiones en Tetela del Volcán son aquellas con algún tipo de privilegio, personas que hayan logrado el respeto comunitario, mujeres con independencia económica, estudios, o una familia que la respalde. "Las que logran acceder a las tomas de decisión tienen que tener trayectoria que las legitima ante el resto de la comunidad. Es decir, van acumulando acciones de participación dentro y fuera de la familia, pero aún es muy influyente su 'calidad moral de buena mujer' es decir que cumpla con las responsabilidades dentro del hogar 'que no descuide sus actividades', y tener participación dentro de la comunidad" (Directora EESTEVOL).

De acuerdo con las fuentes secundarias consultadas, $22 \%$ de las mujeres, además del trabajo doméstico se insertan en el mercado laboral "formal" (INEGI, 2015) y se enfrentan a una división sexual y racial del trabajo, condiciones precarias con nulas prestaciones laborales, así como menores ingresos con respecto a los hombres. Las mujeres $(39,5 \%)$ reciben en promedio menos de 1 salario mínimo mensual (menos de 100 USD), al contrario de ellos $(34,4 \%)$ con 1 a 2 salarios mínimos mensuales (de 100 a 200 USD) (INEGI, 2015).

Durante los recorridos en campo y de acuerdo con información oficial, se confirmó que las afectaciones en las unidades económicas impactaron directamente sobre la autosuficiencia económica de las mujeres, pues $62 \%$ de la población femenina económicamente activa depende del comercio y servicios (INEGI, 2015). A la par, algunas artesanas productoras de licores frutales y mermeladas perdieron sus utensilios de trabajo, así como sus casas que también son los espacios donde elaboran sus productos (Sánchez, 2017).

Considerando las dinámicas de la vida cotidiana, la temporalidad y espacialidad son dimensiones relevantes que se expresaron en las entrevistas y durante las actividades realizadas. Así, con respecto al día y hora del sismo, los hombres se encontraban en el campo y las mujeres en las casas, o en la calle realizando compras para la comida o en camino a las escuelas para recoger a las y los niños. 
Las mujeres embarazadas, niñas y niños pequeños, personas con dificultad de movimiento 0 enfermas y personas de edad avanzada pasan la mayor parte del tiempo dentro de las viviendas, como se observó en la condición de una mujer de 80 años afectada: "Ella vivía totalmente sola, padecía diabetes y apenas podía caminar (...) el terremoto tiró el muro de su habitación directamente sobre su cama; durante el sismo se encontraba trabajando en una tienda de abarrotes en la ventana de su casa como forma de sustento" (Notas de campo personales).

Por otro lado, las informantes pusieron de relieve el control de los varones y las instituciones sobre el cuerpo de las mujeres en temas de salud integral, planificación familiar, violencia física, sexual, emocional y económica. Por ejemplo, los reportes consultados del grupo de Sumando Voluntades (2017), hace énfasis en la problemática de abusos sexuales hacia las mujeres, temática que emergió de manera recurrente durante la participación en las brigadas psicológicas de apoyo postdesastre. La misma problemática se evidenció durante el trabajo en el temazcal: "Una mujer de 60 años nos platicó que el tema de los abusos sexuales era muy común dentro de los hogares y que se repetía de generación en generación. Ella había sido abusada sexualmente por su abuelo, y su madre fue abusada sexualmente por su propio padre" (Notas de campo personales).

Estos delitos son agravados por factores como altos niveles de hacinamiento en los hogares o la ingesta de alcohol por parte de los varones. Así, las mujeres señalan que sus casas se convierten en lugares de alto riesgo, con poca posibilidad de denuncia por la preponderancia en la comunidad de valores centrados en la familia, la dependencia económica, patrimonial y cultural de las mujeres hacia la pareja masculina.

\section{Territorio: Las mujeres al cuidado del cuerpo común, su comunidad}

Los hallazgos en campo muestran que las jornadas de trabajo (remunerado y no remunerado) de las mujeres se duplicaron o triplicaron en los días posteriores al sismo, en actividades de reconstrucción, labores de cuidados, trabajo asalariado, trabajo comunitario y cuidado de infantes por el cierre de las escuelas. No obstante, las mujeres se organizaron para responder a necesidades no satisfechas o no percibidas, principalmente obligaciones del rol materno tradicional (Neal \& Phillips, 1990) y que desde nuestra perspectiva tiene que ver con los cuidados diferenciados que los miembros de la comunidad brindan, los cuales en momentos de emergencia se hacen evidentes junto con la solidaridad y la revitalización de los lazos comunitarios.

Se documentó que un gran número de mujeres de la comunidad salieron a las calles con enormes recipientes de comida para brindar alimento a la población sin hogar, junto con los voluntarios y voluntarias que acudieron de diversas partes del país y del extranjero para coadyuvar con las tareas de recuperación del sismo. A la par, en la sede de la EESTEVOL se instaló un comedor comunitario conformado por un grupo de mujeres de la comunidad que trabajaron directamente en la preparación de alimentos. Algunas mujeres de forma independiente generaron vínculos con personas externas a la comunidad solicitando donaciones, otras más lideraron grupos de trabajo establecidos en diferentes sedes como el palacio municipal, iglesias o la universidad.

Durante las visitas a campo se observó la promoción del trabajo comunal de la directora de la EESTEVOL, al organizar un centro comunitario de atención a la emergencia en esta escuela. Es interesante notar cómo esta mujer originaria de la comunidad, fue legitimada para gestionar, coordinar y atender necesidades de afectados, voluntarios, brigadistas y autoridades locales (comisariados ejidales y comunales, representantes de barrio y de diversos grupos de la comunidad): "Esto ha sido difícil ... ser mamá, trabajadora y profesionista no ha sido sencillo, es un esfuerzo físico, emocional y un proceso de ruptura de paradigmas. Esto me dio fortaleza ante la comunidad y la familia. Tuve que romper con el propio ciclo de vida, con una desobediencia a las normas" (Directora EESTEVOL).

Otro aspecto a destacar en los hallazgos, se refiere al acompañamiento emocional, una actividad considerada como femenina, que funciona a través del acuerpamiento como forma de contención de dolor, una mujer de Tetela comentó "yo, la verdad prefiero estar aquí cocinando y ayudando en lo que puedo, que adentro de mi casa" (Nota de campo personal, Mujer 35 años).

\section{DISCUSIÓN}

Entender la vulnerabilidad socioambiental con las contribuciones del marco analítico de los feminismos comunitarios, permite nombrar y entender desde una perspectiva diferente las opresiones que se ejercen sobre el cuerpo-territorio y territorio-tierra, como un sistema que se 
teje históricamente sobre el cuerpo de las mujeres en la comunidad de estudio. Así, uno de los hallazgos en esta investigación, reafirma que las mujeres empujan sus luchas desde el trabajo comunal (Tzul, 2015) a través de prácticas cotidianas para recuperar el espacio donde se llevan a cabo las interacciones sociales: su comunidad.

La conceptualización del entronque patriarcal ejemplifica la complejidad en Tetela del Volcán, una comunidad campesina influenciada por el colonialismo occidental y el patriarcado moderno, estructuras que se reproducen a través de las instituciones, los medios de comunicación y los símbolos culturales (Cabnal, 2012). Asimismo, como producto de las condiciones históricas es posible identificar en Tetela un patriarcado ancestral (Cabnal, 2012) que aparece en las tradiciones indígenas Nahuas y se manifiesta en las costumbres, la relación sociedad-naturaleza y las formas de gobierno dominantes entre otras prácticas.

En este sentido, la complejidad socioambiental de localidades como Tetela del Volcán se expresa en el carácter ambivalente de la religión, las tradiciones, la constitución nuclear de familia y el control de la tierra mediante lazos de parentesco, dado que funcionan como organizadores sociales encargados de reproducir las relaciones de poder sobre las mujeres; al mismo tiempo se erigen como formas de articulación, organización, cohesión de la comunidad y defensa del territorio. Elementos que constituyen el sistema de gobierno comunal indígena (Tzul, 2015), el cual actúa como estrategia para autorregular y defender su territorio, así como un piso donde se reproduce el trabajo comunal de hombres y mujeres.

La Comunidad (Paredes \& Guzmán, 2014) es la integración de la diversidad, no solo replicable en contextos rurales, sino en todo tipo de territorialidades, marca el camino de la emancipación de hombres, mujeres y sus territorios para la lucha por el buen vivir, el cual tiene el potencial de representar el espacio de acción desde el que es posible la transformación social, sobre todo a partir de procesos de organización y participación conjunta.

Las resistencias y las comunalidades de las mujeres resuelven situaciones de desastre, tejiendo lazos solidarios y reconstruyendo socialmente sus comunidades. Dada la anulación como sujetas políticas por algunos discursos hegemónicos, la capacidad organizativa de las mujeres como respuesta al sismo, mediante la atención de problemáticas cotidianas requiere visibilizarse. La experiencia en Tetela del Volcán muestra procesos endógenos, en los cuales se identifica a aquellas mujeres que por su reconocimiento en la comunidad, sus redes sociales y legitimación en la estructura social son capaces de resumir y recoger saberes de la comunidad (Gargallo, 2014).

Las políticas internacionales para la reducción del riesgo y la vulnerabilidad de mujeres, que se implementan en México (INMUJERES, 2013) desde la perspectiva de los feminismos hegemónicos europeos, promueven acciones como el empoderamiento, liderazgo, equidad de género, la participación en la toma de decisiones y la promoción de las mujeres como agentes del desarrollo. Sin embargo, los datos de campo demuestran que, en Tetela del Volcán, la realidad de mujeres campesinas/indígenas es más compleja que las propuestas generalizadoras de la realidad provenientes de estas organizaciones.

Desde la perspectiva del feminismo comunitario, la apuesta es por la recuperación de la dualidad cosmogónica (dualidad no binaria, ni entendida como pareja heterosexual) como dualidad política (hombre-mujer) que rige el equilibrio, en oposición a la idea de 'igualdad de género'; para construir de las diferencias una sociedad diversa y solidaria.

Los valores de confianza y reciprocidad que se observaron en la población de Tetela para enfrentar la tragedia y el dolor, son los mismos que constituyen el trabajo comunitario para las faenas (reforestación, limpieza) y en la organización de sus festividades (día de muertos o la semana santa), para lo cual se cuenta con la participación de cada uno de los integrantes como una responsabilidad y compromiso con la comunidad:

"Cuando llegué a Tetela empaticé con la comunidad al ver el dolor (...) también algo que llamó mi atención (...) la enorme gratitud de las personas que padecieron un desastre hacia los que 'íbamos a ayudarles' compartiendo alimento, agradeciendo con carteles, brindando hospedaje (Notas de campo personales)."

Los eventos de crisis social y/o ambiental pueden contribuir a romper con el orden de género previo, movilizando energías y resistencias relacionadas con derechos sobre la tierra, la salud, el 
duelo, la participación en la reconstrucción y la vida social en general (Fernández et al., 2020). Sin embargo, son muy evidentes las violencias hacia las mujeres como efecto del patriarcado y del colonialismo interno que sirve de base racista para la implementación de políticas de ajuste neoliberal (Paredes, 2008) que perpetúan las desigualdades en cuerpos generizados y racializados.

En Tetela del Volcán, las violencias se revelaron como consecuencia inmediata del terremoto del 19S, por ejemplo, las dos mujeres que perecieron compartieron la imbricación de tres ejes de opresión: provenían de raíces campesinas o indígenas (racismo); eran mayores de 50 años solteras, situación que las coloca con mayor vulnerabilidad socioeconómica por las dinámicas de la comunidad y se encontraban en sus viviendas (patriarcado); tercero, es posible afirmar que por la precariedad de sus viviendas se encontraban en situación de pobreza.

La lógica de las prácticas comunitarias campesinas/indígenas expresa la prevalencia y conjunción de los patriarcados ancestral y moderno. Así, las mujeres forman su propia familia recreando la comunidad y al mismo tiempo la unidad familiar se beneficia de diversas formas en lo individual y en lo social. Aunque también se expresan las violencias que transforman en objeto a las mujeres, desde la naturalización de las agresiones en el cuerpo, la posesión del hombre sobre la mujer, la expectativa de obediencia y sumisión, entre algunos atributos para ser y pertenecer al núcleo familiar y por tanto a la comunidad, ejerciendo con ello violencias múltiples que se proyectan en el sistema cuerpo-territorio-tierra.

Con los factores de contexto arriba enunciados -la vulnerabilidad socioambiental de Tetela del Volcán, la vulnerabilidad de las mujeres y el trabajo comunal para la atención de la emergenciaencontramos en los feminismos comunitarios ejes de análisis que pueden acompañar la lucha contra la dominación sobre los cuerpos, los territorios y por consiguiente para la disminución en la vulnerabilidad a desastres.

No es nuestra intención asegurar que existe un feminismo comunitario en la localidad, tales enunciaciones corresponden a las mujeres de Tetela del Volcán, lo que sí logramos es nombrar pistas para entender la racionalidad de las acciones colectivas y plantear los retos que el marco analítico de los feminismos comunitarios propone para el análisis de la vulnerabilidad a desastres. En este sentido, establecer diálogos con epistemologías diversas coadyuva en la generación de enfoques teóricos, conceptuales, metodológicos, así como propuestas que provienen de las y los sujetos que habitan este espacio. En particular, desde el marco analítico de la vulnerabilidad socioambiental a desastres, ignorar otros pensamientos fuera de las lógicas occidentales influye en el abordaje y toma de decisiones sobre los procesos postdesastre, los cuales fortalecen enfoques cientificistas eurocéntricos que privilegian un modelo de sociedad ajeno al que se vive en el Sur.

\section{REFLEXIONES FINALES}

Los vacíos teóricos en las ciencias de los desastres se reflejan en la ausencia de una teoría de vulnerabilidad socioambiental construida con la perspectiva del Sur, de las mujeres que sufren opresiones, así como las resistencias diversas que expresan el lugar y el sujeto desde el cual se enuncian. La colonización epistemológica (Santos, 2010) en la que las y los científicos latinoamericanos estamos inmersos nos despoja de la imaginación necesaria para construir marcos analíticos y categorías acordes a nuestra realidad. Por ello, ante la diversidad y complejidad de cada grupo social se tienen que construir estrategias desde la voz de quienes padecen las vulnerabilidades, en este caso, las mujeres junto con los hombres, en construcción paralela para superar las formas fraccionadas de leer la realidad (Cumes, 2012).

El caso del sismo de 2017 en Tetela de Volcán, abordado desde el lente de los feminismos comunitarios, evidencia no solo las prácticas patriarcales y coloniales de largo alcance en el cual las mujeres son excluidas de bienes materiales y espacios de decisión, sino también los vacíos epistémicos y metodológicos que aún predominan para comprender y atender los impactos de un desastre.

La incorporación del feminismo hegemónico o de la igualdad en las ciencias de los desastres, despolitiza la perspectiva feminista, universaliza la condición de la mujer y propone medidas ajenas a la realidad de las comunidades en América Latina, como el empoderamiento femenino o la reconstrucción para el desarrollo, basados en la aplicación de medidas que corresponden a lógicas diferentes. Se ignoran otros feminismos basados en lógicas duales y del buen vivir, desplazando a las mujeres como sujetos políticos (Curiel \& Galindo, 2015). 
Se trata de desafiar las estructuras, lógicas y sentidos de las instituciones públicas de control del poder (Galindo, 2013), de no hacerlo, solo incluiremos la perspectiva de género para reconocer identidades con base en el género, raza, edad, sexualidad y su vulnerabilidad ante los desastres, más que el cuestionamiento del mundo que ha dado lugar a que ser diferentes, signifique ser desiguales (Cumes, 2012). Pensar en conceptos como dualidad, equilibrio, armonía (Marcos, 2014) marcan las pistas para la generación de una teoría crítica de la vulnerabilidad socioambiental, desde las mujeres de América Latina y el Caribe que proponga otras formas de conocimiento e interpretación de la realidad, como las filosofías o cosmogonías indígenas para entender el desastre, la vulnerabilidad socioambiental y las apuestas emancipatorias.

Este ejercicio analítico pretende ser una provocación para la reflexión académica, con la intención de influenciar las decisiones políticas y las agendas científicas con las experiencias y sabidurías locales. Esto supone un reto en el ámbito académico porque requiere un proceso de descolonización científica, con una apertura a epistemologías alternativas como las que han desarrollado los pueblos originarios de América Latina, con una ciencia comprometida y de largo alcance, que se extienda además en las prácticas políticas y que abone a procesos autogestivos. En este sentido, las propuestas de los feminismos comunitarios resultan pertinentes para el abordaje de manera integral y profunda de los procesos socioambientales constitutivos del desastre.

\section{REFERENCIAS}

Acuña, A. (2016). El temazcal en Santa María Yucunicoco (Oaxaca, México): Un lugar para la recreación del cuerpo y la sociedad. Revista de Antropología Experimental, 16, 45-61. https://doi.org/10.17561/rae. voi16.3131

ARP Prensa. (2017, septiembre 20). Parte informativo por la alcaldesa de Tetela del Volcán tras sismo [Archivo de video]. Recuperado de_https://youtu.be/1SkaqFx1678

Bazaldúa, B. \& García, A. (2014). Factores que limitan la eficiencia de organizaciones productoras de aguacate: el caso del municipio de Tetela del Volcán, Morelos. Entreciencias: diálogos en la Sociedad del Conocimiento, 4(2), 183-196. http://dx.doi.org/10.21933/J.

EDSC.2014.04.082

Berrio, L. (2019). Conversaciones a tres voces sobre el feminismo comunitario de Guerrero. Encartes, 2(4), 232-247. https://doi.org/10.29340/en.v2n4.93

Barranco, A. (17 de septiembre de 2018). Informe de viviendas afectadas por el sismo del 19 de septiembre del 2017 y zonas de riesgo geológico y ambiental. Manuscrito no publicado, Tetela del Volcán, Morelos, México.

Blaikie, P., Cannon, T., Davis, I. \& Wisner, B. (1994). At Risk: Natural Hazards, People's Vulnerability and Disasters. Londres: Routledge.

Bradshaw, S. (2013). Gender, development and disasters. England: Edward Elgar Publishing.

Bradshaw, S., \& Arenas, Á. (2004). Análisis de género en la evaluación de los efectos socioeconómicos de los desastres naturales. Santiago de Chile: Naciones Unidas, CEPAL.

Bravo, E. (2017). El sismo del 16 de abril en Manabí visto desde la Ecología Política del desastre. Universitas, XV(26), 235-252. https://doi.org/10.17163/uni.n26.2017.10.

Cabnal, L. (2012). Feminismos diversos: el feminismo comunitario. España: ACSUR-Las Segovias.

Cabnal, L. (2017). Tzk'at. Red de sanadoras ancestrales del feminismo comunitario desde IximulewGuatemala. Ecología Política (54), 98-102. http://www.jstor.org/stable/44645644

Cabnal, L. (2017, septiembre 21). Lorena Cabnal en las II Jornadas de investigaciones feminista y de género. [Archivo de video]. Recuperado de https://youtu.be/uoj6j4kTLqQ

Cabral, V. \& Zulaica, L. (2015). Análisis de la vulnerabilidad socioambiental en áreas del periurbano de Mar de Plata (Argentina) expuestas a agroquímicos. Multiciencias, 15(2), 172-18o. http://hdl.handle. net/11336/62432

Castañeda, M. (2012). Etnografía feminista. En Blazquez, N., Flores, F. \& Ríos, M. (coord.). Investigación feminista: Epistemología, metodología y representaciones sociales (pp. 217-238). México: UNAM.

Castro, C. \& Reyes, L. (2006). Desastres naturales y vulnerabilidad de las mujeres en México. México: INMUJERES, PNUD.

Centro Nacional de Prevención de Desastres (CENAPRED). (2019). Impacto socioeconómico de los principales desastres ocurridos en la república mexicana en el año 2017. México: Dirección de difusión CENAPRED 
Comisión Nacional de Evaluación de la Política de Desarrollo Social (CONEVAL). (2015). Pobreza a nivel municipio 2015. CONEVAL, México. Recuperado de: https://www.coneval.org.mx/coordinacion/ entidades/Morelos/Paginas/pobreza_municipal2015.aspx

Corbetta, P. (2003). Social research: Theory, methods and techniques. Sage.

Cueto, R., Seminario, E., \& Balbuena, A. (2015). Significados de la organización y participación comunitaria en comunidades vulnerables de Lima Metropolitana. Revista de Psicología (PUCP), 33(1), 57-86. http://www.scielo.org.pe/scielo.php?script=sci_arttext\&pid=So254-92472015000100003\&lng= es\&tlng=es

Cumes, A. (2012). Mujeres indígenas patriarcado y colonialismo: un desafío a la segregación comprensiva de las formas de dominio. Anuario de Hojas de WARMI, (17).

Curiel, O. (2014). Construyendo metodologías feministas desde el feminismo decolonial. En Mendia, I., Luxán, M., Legarreta, M., Guzmán, G., Zirion, I. \& Azpiazu, J. (eds.). Otras formas de (re)conocer, Reflexiones, herramientas y aplicaciones desde la investigación feminista (pp. 24-44). España: Hegoa.

Curiel, O. \& Galindo, M. (2015). Descolonización y despatriarcalización de y desde los feminismos de Abya Yala. Catalunya: ACSUR-Las Segovias.

Curiel, O. [CICODE UGR]. (2017, enero 19). Ochy Curiel. Feminismo Decolonial. Prácticas Políticas Transformadoras. Conferencia [Archivo de video]. Recuperado de https://youtu.be/BovLlIncsgo

Enarson, E. \& Morrow, B. (1998). Why gender? Why women? An introduction to women and disaster. En Enarson, E. \& Morrow, B. The gendered terrain of disaster: Through women's eyes. EE.UU.: Praeger Press.

Escobar, A. (2007). La invención del Tercer Mundo. Construcción y deconstrucción del desarrollo. Venezuela: Fundación Editorial el perro y la rana.

Espinosa, Y. (2014). Una crítica descolonial a la epistemología feminista crítica. El Cotidiano, (184), 7-12. https://www.redalyc.org/articulo.oa?id=325/32530724004

Fernández, A., Waldmüller, J., \& Vega, C. (2020). Comunidad, vulnerabilidad y reproducción en condiciones de desastre. Abordajes desde América Latina y el Caribe. Íconos. Revista de Ciencias Sociales, (66), 7-29. https://doi.org/10.17141/iconos.66.2020.4156

Fini, D. (2016). Territorio como paradigma en las luchas sociales contemporáneas. Navarro, M. \& Fini, D. (coords.). Despojo capitalista y luchas comunitarias en defensa de la vida en México. Claves desde la ecología política (pp.93-110). México: Instituto de Ciencias Sociales y Humanidades "Alfonso Vélez Pliego", Benemérita Universidad Autónoma de Puebla.

Gaillard, J., Gorman, A. \& Maureen, F. (2017). Sexual and gender minorities in disaster. Sexual and gender minorities in disaster, Gender, Place E Culture, 24(1), 18-26. https://doi. org/10.1080/0966369X.2016.1263438

Galindo, M. (2013). No se puede descolonizar sin despatriarcalizar. Teoría y propuesta de la despatriarcalización. Bolivia: Mujeres Creando.

Gargallo, F. (2014). Feminismos desde Abya Yala. Ideas y proposiciones de las mujeres de 607 pueblos en nuestra América. México: Corte y Confección.

Gargallo, F. (2016). Consolidación de las ideas y prácticas feministas latinoamericanas: del feminismo de la igualdad al feminismo comunitario. Revista venezolana de estudios de la mujer, 21(46), 33-45. ISSN 1316-3701

Geertz, C. (2008). Thick description: Toward an interpretive theory of culture. Londres: Routledge.

Gobierno del Estado de Morelos. (2017). Estatus de afectaciones por el sismo del 19 de septiembre de 2017. Unidos por Morelos. Recuperado de: http://unidospormorelos.morelos.gob.mx/

González, J. (2019). Percepciones sobre el territorio y riesgos socioambientales con perspectiva de género, en la localidad de Tetela del Volcán, Morelos, México. Morelos, México: Centro Regional de Investigaciones Multidisciplinarias, UNAM.

Hewitt, K. (ed.). (1983). Interpretations of calamity: From the viewpoint of human ecology. EE.UU.: Allen \& Unwin Inc.

Hill, P. (1998). Fighting words. Black women and the search for justice. EE.UU.: University of Minnesota.

Instituto Nacional de Estadística y Geografía (INEGI). (2015). Tabulados de la encuesta intercensal 2015. Recuperado de https://www.inegi.org.mx/programas/intercensal/2015/ 
Instituto Nacional de las Mujeres (INMUJERES). (2013). Equidad de género en la gestión integral del riesgo. Programa de capacitación "Protección civil y Gestión integral del riesgo asociado a casos de desastres. México: INMUJERES, SINAPROC.

Lander, E., \& Castro, S. (200o). La colonialidad del saber: eurocentrismo y ciencias sociales: perspectivas latinoamericanas. Buenos Aires: Consejo Latinoamericano de Ciencias Sociales-CLACSO.

Lugones, M. (2008). Colonialidad y género. Tabula rasa, (o9), 73-101. https://revistas.unicolmayor.edu. co/index.php/tabularasa/article/view/1501

Maldonado, N. (2007). Sobre la colonialidad del ser: contribuciones al desarrollo de un concepto. El giro decolonial. Reflexiones para una diversidad epistémica más allá del capitalismo global, 127-167.

Marcos, S. (2014). La espiritualidad de las mujeres indígenas mesoamericanas: descolonizando las creencias religiosas. En: Espinosa, Y., Gómez, D. \& Ochoa, K.(eds.). Tejiendo de otro modo: Feminismo, epistemología y apuestas descoloniales en Abya Yala (pp.143-159). Colombia: Editorial Universidad del Cauca.

Martínez, E. (2008). Las relaciones global-local en la transformación socioespacial de la región de los Altos de Morelos en México. Secuencia (70),129-166. https://doi.org/10.18234/secuencia.voi70.1033

Moore, C. (2018). Feminismos del Sur, abriendo horizontes de descolonización. Los feminismos indígenas y los feminismos comunitarios. Estudios Políticos (53), 237-259. http://doi.org/10.17533/ udea.espo.n53a11

Neal, D. \& Phillips, B. (1990). Female-dominated local social movement organizations in disaster-threat situations. En: West, G. \& Blumberg, R. Women and social protest. NY, EE.UU.: Oxford University Press.

Paredes, J. (2008). Plan de las mujeres: marco conceptual y metodológico para el Buen Vivir. Revista de estudios Bolivarianos, 15-17, 191-210. http://doi.org/10.5195/bsj.2010.9

Paredes, J. (2013). Hilando fino desde el feminismo comunitario. México: Cooperativa El Rebozo, Zapateándole, Lente Flotante, En cortito que’s palargo y AliFem AC.

Paredes, J. (2017). El feminismocomunitario: la creación de un pensamiento propio. Corpus [En línea], 1(7). http://corpusarchivos.revues.org/1835

Paredes, J. \& Guzmán, A. (2014). El tejido de la rebeldía: ¿Qué es el feminismo comunitario?. La Paz, Bolivia: Comunidad Mujeres Creando Comunidad.

Programa de las Naciones Unidas para el Desarrollo (PNUD). (2007). Superar la desigualdad, reducir el riesgo. Gestión del riesgo de desastres con equidad de género. México: Programa de las Naciones Unidas para el Desarrollo.

Quijano, A. (200o). Colonialidad del poder, eurocentrismo y América Latina. En Lander, E. (comp.) La colonialidad del saber: eurocentrismo y ciencias sociales.Perspectivas latinoamericanas. Buenos Aires: CLACSO.

Reyes, M. (2017). Capital social y acción colectiva en la autogestión social del agua: manguereros y cajas de agua en Tetela del Volcán. [Tesis de doctorado]. Cuautla, Morelos: Universidad Nacional Autónoma del Estado de Morelos.

Reyes, M. (2011). La organización local y los recursos de uso común en Tetela del Volcán. Actores, espacios de decisión y sistemas de gobierno. [Tesis de maestría]. Cuernavaca, Morelos: Instituto Profesional de la Región Oriente, Universidad Autónoma del Estado de Morelos.

Rojas, L. (coord.). (2015). Neoliberalismo en América Latina.Crisis, tendencias y alternativas. Asunción: CLACSO.

Romero, H., \& Romero, H. (2015). Ecología política de los desastres: vulnerabilidad, exclusión socioterritorial y erupciones volcánicas en la Patagonia chilena. Magallania (Punta Arenas), 43(3), 7-26. http://dx.doi.org/10.4067/So718-22442015000300002

Sánchez, M. (10 de octubre de 2017). Terremoto destruyó economía local de Tetela de Volcán. Sin Línea Diario. Recuperado de https://sinlineadiario.com.mx/ terremoto-destruyo-economia-local-de-tetela-del-volcan/

Serrano, R. (2013). La observación participante como escenario y configuración de la diversidad de significados. En Tarrés, M. (coord.). Observar, escuchar y comprender. Sobre la traducción cualitativa en la investigación social (pp. 93-124). México: FLACSO.

Santos, B. (2010). Descolonizar el saber, reinventar el poder. Montevideo: Ediciones Trilice. 
Stake, R. (2005). Qualitative Case Studies.En Denzin, N. \& Lincoln, Y. (eds.)The Sage handbook of qualitative research ( $5^{a}$ Ed.) (pp.443-466). San Francisco:SAGE Publications.

Strauss, A., \& Corbin, J. (2016). Bases de la investigación cualitativa: técnicas y procedimientos para desarrollar la teoría fundamentada. Medellín: Universidad de Antioquia.

Sumando Voluntades. (2017). Primer informe del Centro Comunitario de Acopio y Logística Regional Tetela del Volcán. Tetela del Volcán, México: Manuscrito no publicado.

Sun, R., Gao, G., Gong, Z. \& Wu, J. (2019). A review of risk analysis methods for natural disasters. Natural Hazards. http://doi.org/10.1007/s11069-019-03826-7

SEDESOL. (2012). Atlas de riesgos naturales de Tetela del Volcán, Morelos, 2012. México: Secretaría de Desarrollo Social.

Segato, R. (2014). El sexo y la norma: frente estatal, patriarcado, desposesión, colonidad. Revista Estudos Feministas, 22(2), 593-616. https://doi.org/10.1590/So104-026X2014000200012

Servicio Sismológico Nacional (SSN). (2017). Reporte especial. Sismo del día 19 de septiembre de 2017, Puebla-Morelos (M 7.1). Recuperado de http://www.ssn.unam.mx/sismicidad/reportesespeciales/2017/SSNMX_rep_esp_20170919_Puebla-Morelos_M71.pdf

Tzul, G. (2015). Sistemas de gobierno comunal indígena: la organización de la reproducción de la vida. El Apantle, 1, 125-141. https://www.jstor.org/stable/j.ctvtxw2km.7

Varela, R. (1985). Estructuras de poder en Morelos. Relaciones, 6(21), 5-33. https://www.colmich.edu. $\mathrm{mx} /$ relaciones25/files/revistas/021/RobertoVarela.pdf

Wisner, B., Blaikie, P., Cannon, T., \& Davis, I. (2004). At risk: natural hazards, people's vulnerability and disasters. NY, EE.UU.: Psychology Press.

Yadav, P., Saville, N., Arjyal, A., Baral, S., Kostkova, P., \& Fordham, M. (2021). A feminist vision for transformative change to disaster risk reduction policies and practices. International Journal of Disaster Risk Reduction, 54. https://doi.org/10.1016/j.ijdrr.2020.102026

Zanella, M., Olímpio, J., Lustosa, M. \& Wenderley, E. (2013). Vulnerabilidade socioambiental do baixo curso da bacia hidrográfica do Rio Cocó, Fortaleza-CE-. Sociedade E Natureza, 25(2), 317-331. http:// dx.doi.org/10.1590/S1982-45132013000200009 